\title{
Pushing the Frontiers of Accessible Chemical Space to Unleash Design Creativity and Accelerate Drug Discovery
}

\author{
Thomas C. Fessard*, Kristina Goncharenko, Quentin Lefebvre, and Christophe Salomé
}

\begin{abstract}
In highly competitive research environments, the ability to access more complex structural spaces efficiently is a predictor of a company's ability to generate novel IP-protected small molecule candidates with adequate properties, hence filling their development pipelines. SpiroChem is consistently developing new synthetic methodologies and strategies to access complex molecular structure, thereby facilitating and accelerating small molecule drug discovery. Pushing the limits of what are perceived as complex molecular structures allows SpiroChem and its clients to unleash creativity and explore meaningful chemical spaces, which are under-exploited sources of novel active molecules. In this article, we explain how we differentiated ourselves in a globalized R\&D environment and we provide several snapshots of how efficient methodologies can generate complex structures, rapidly.
\end{abstract}

Keywords: C-H activation · Late-stage functionalization · Synthetic efficiency $\cdot$ sp3 scaffolds

\section{Business Case, Context and Motivation}

\subsection{The Renewed Importance of Synthetic Chemistry in Drug Discovery at the Dawn of the $21^{\text {st }}$ Century}

\subsubsection{From a Scientific Point of View: The Need for Innovation}

In the last three decades, the drug discovery model has been influenced by the rapid advances and breakthroughs in biology, both in the understanding of diseases and in the techniques used for screening. This has shone a dazzling light on biology and made it the most visible provider of innovation in the Life Sciences. It also raised hopes that one day biology alone would help cure most, if not all, diseases. This (justified) praise of the rapid evolution of biology indirectly coined synthetic organic chemistry as an 'old' science where innovation is scarce and that delivers molecules when asked, no matter where or by whom the chemistry would be performed. With this idea that every chemist can "do the job" and that quantity equals quality, the industry started to outsource massively its chemistry R\&D to places where costs of operations are reduced. As we now see a posteriori, this led to reduced productivity and innovation, loss of internal capabilities and critical mass to tackle new problems, thereby confirming the self-fulfilling prophecy that chemistry is outdated (in the eyes of management).

Ironically, in the same period, some voices in industry started to plead in favor of the introduction of more sp3 character and reduction of the aromatic ring counts as a way to improve the properties and chances of success of development candidates. ${ }^{[1]}$ Unfortunately, increasing the number of $\mathrm{sp} 3$ centers in a molecule generally correlates with an increase of synthetic complexity and can be seen as a hurdle for those who have to design a proper Structure-Activity Relationship study. Therefore, medicinal chemists got stuck between two opposite trends: the increasing complexification of the designed molecular targets and the decreasing resources that would allow these molecules to be studied efficiently.

${ }^{*}$ Correspondence: Dr. T. C. Fessard, E-mail: thomas.fessard@spirochem.ch SpiroChem AG, Mattenstrasse 24, CH-4058 Basel, Switzerland
In the meantime, academic research in organic chemistry has renewed itself, especially in the reaction methodology field, shifting from a 'how to make things perfect' approach (anything below 99:1 e.r. is not worth publishing) to a 'how to make things different' approach. The field has even witnessed an acceleration of this trend in the last 10 years, with the development of techniques and methodologies that focused on reaction discovery and new transformations, allowing $\mathrm{C}-\mathrm{H}$ activation and late-stage functionalization of positions that were previously seen as unreactive. These advances can serve to explore new territories of structural space and unleash the creativity of designers by removing the constraints of perceived chemical complexity and limited accessibility.

Of course, there is a delay in adoption of new techniques by industry, especially for companies who do not have in-house wet lab capabilities or are over-dependent on service providers that are unable to incorporate efficiently these new techniques.

In this context, the mission and important role of SpiroChem in the industry is to serve as an accelerator of innovation and an interface between academia (solutions) and industry (needs).

\subsubsection{From a Business Point of View: The Need for Speed}

The scientific (r)evolutions in biology mentioned above often emerge from universities or research centers and require less infrastructure for implementation than traditional discovery, making them accessible to smaller research entities (i.e. startups) who are usually less risk adverse and more agile when it comes to implementing new technologies or concepts. This has led the Life Science industry to a new R\&D paradigm, where large portions of the innovation and discovery field are now performed outside traditional big pharma companies, mainly in biotech companies. These biotech companies are often lean (compared to pharma) and even virtual. This has also profoundly changed the financial model of drug discovery, where investors (Business Angels, VCs) expect returns on investment in timelines that are are much shorter than large organizations. The time and efforts between each value inflexion point, i.e. advances in the project such as POC in animal, candidate selection, first-in-man studies are directly impacting the burn rate of the company and its financial stability. Delays may force small companies to seek interim refinancing (resulting in 
dilution of their existing shareholders) and in extreme cases cause their bankruptcy. SPEED of R\&D is therefore as important as scientific soundness, and any way of accelerating/shortening each stage in the discovery pipeline means increased odds of success.

\subsection{Reconciliating Speed and Innovation to Accelerate $R \& D$ and Reduce Overall Discovery Costs}

When attrition is factored in, about one third of the total budget to bring a new medicine to the market (currently estimated at $\$ 1.5 \mathrm{bn}+$ ) is spent at the discovery stage, i.e. before a final development candidate has been selected. The ability to explore new chemical space, and make the 'right' molecule, irrespective of its perceived synthetic complexity, in a short and efficient manner should result in a shortening of the optimization cycles and, when coupled to an efficient design rationale, reduce the number of optimization cycles required. This would in turn reduce the overall costs of discovery and deliver candidates to the clinic in an accelerated manner.

\subsection{SpiroChem: Historical Development and Positioning}

SpiroChem was founded in 2011 as an ETH-Zurich spin-off, with the concept of providing advanced building blocks with beneficial intrinsic properties such as ADME (Absorption, Distribution, Metabolism, Excretion) or new IP (Intellectual Property) to discovery chemists and therefore save them time. SpiroChem faced a challenge though: doing fine chemistry in a high-cost country (Switzerland) would be financially sustainable only if 1) the molecules we made had very high added value and 2) we could make them efficiently. So early on, SpiroChem had to focus on innovation, top-notch human resources and efficient work-flows to stay afloat and grow. Innovation not only concerned the chemical space we covered, but how those molecules could be accessed (synthetic accessibility) and used (medicinal chemistry relevance) (Fig. 1).

Our product offering evolved to SpiroKits ${ }^{\mathrm{TM}},{ }^{[2]}$ focused libraries and unique libraries of fragments/scaffolds for DELt (DNAEncoded Library Technology) (Fig. 2).

\subsection{Transitioning from Service to Solution Provider}

As demonstrated earlier, chemistry capabilities in large traditional organizations have shrunk while new discovery players remain relatively small to retain swiftness or due to limiting funding. This has led the industry to rely on outsourcing to Contract Research Organizations (CROs), and by management/shareholder pressure, to low-cost CROs. This intrinsically limits the ability to rapidly introduce and implement new techniques and concepts

In this context, we decided to take the opposite direction by constantly developing new strategies to introduce these new concepts and tools for medicinal chemistry at a late stage, irrespective of the complexity of the motif. By proactively designing at the same time the tools and methods to use them, we reduce the downstream R\&D efforts for our partners and clients. The com-

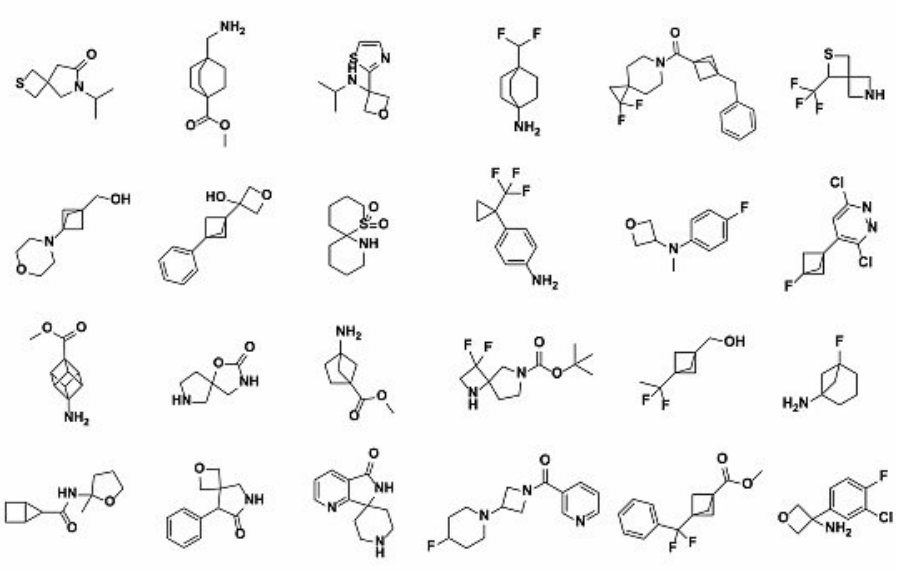

Fig. 2. Selected examples from SpiroChem's unique fragments collection.

pendium of synthetic solutions thus created becomes a toolbox of opportunities to accelerate medicinal chemistry programs: we decorrelate structural complexity from synthetic accessibility.

The years of constant optimization of our workflows and internal procedures to survive and grow in the catalog/product business have generated such efficiency gains that, when applied to contract R\&D, it tremendously benefits our projects, our partners and our clients.

SpiroChem is committed to pushing the frontiers of chemistry, and is also building bridges with other disciplines such as computational chemistry, machine learning ${ }^{[3]}$ or quantum chemistry ${ }^{[4]}$ to further explore and map the structural-property space using modern tools.

\section{Selected Examples of Rapid Synthesis of Complex sp2-sp3 Motifs and Late-Stage Functionalization}

Snapshot 1: Heterocyclic sp2-sp3 building blocks offer improved pharmacokinetic properties over more classical, 'flat' scaffolds. Specifically, bicyclo- and spiroalkanes are sp3-rich (more 3D-like), conformationally-restricted and strained building blocks that are under-represented in major collections despite their high values to medicinal chemists. Their synthesis comes with additional challenge.

Bicyclo[x.y.z]alkanes in particular are of interest to medicinal chemists. They are sp3-rich moieties with advantageous physical and chemical properties and recently their use in medicinal chemists increased as bioisosteres of aromatic ring ${ }^{[5]}$ or alkyne. ${ }^{6]}$ This family of compounds is usually accessible from the parent carboxylic acid. To quickly access sp2-sp3 building blocks, in a step-economical manner, we decided to use Minisci reactions, a well-known selective decarboxylative heteroarylation. ${ }^{[7]}$ The principle of this reaction is the addition of a free radical onto an heteroaromatic system (mostly electron poor). The radical is
Fig. 1. SpiroChem evolution of business offerings.

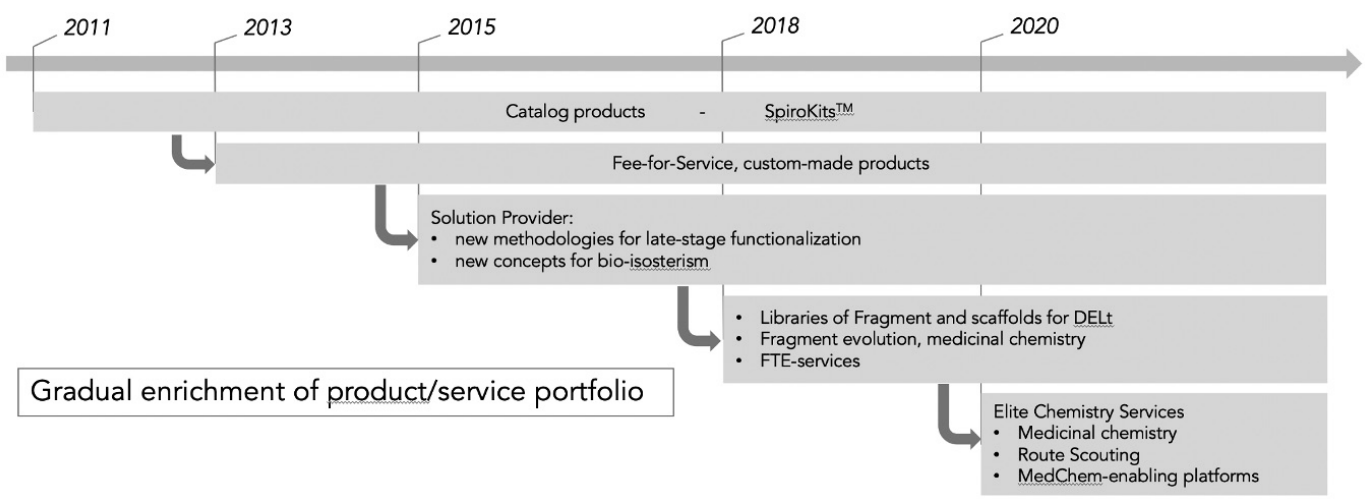


typically formed by oxidative decarboxylation using silver salts and an oxidizing agent. A range of bicycloalkane carboxylic acids were added to heteroaromatic in medium yields (9-63\%). A variety of functional groups are tolerated, such as ester, ketone or nitrile. These functional groups offer additional opportunities for further derivatizations (Scheme 1).

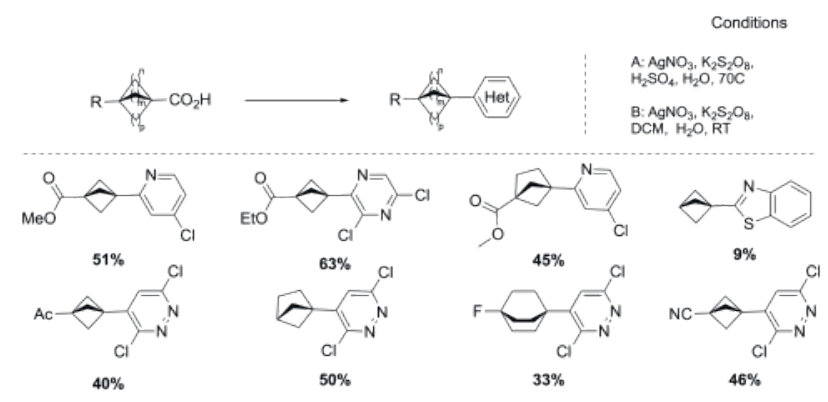

Scheme 1. Synthesis of fused heterocycle/bicyclopentane via the Minisci reaction.

Snapshot 2: The development of new concepts and/or new moieties (such as oxetanes or bicylopentanes) requires the development of innovative new methodologies or tools. At SpiroChem, we endeavor to develop new reaction conditions in order to facilitate the adoption of the novel building blocks that we are creating by the medicinal chemistry community. For instance, oxetanyl amines are important isosteres of amides developed by Carreira $e t$ al. ${ }^{[8]}$ but their reactivity and stability are very different compared to amines or amides and prevent classical $N$-arylation reactions: classical conditions did not provide the desired products. In our efforts to build new sp2-sp3 fragments, we aimed at developing new conditions for $\mathrm{N}$-arylation of oxetanyl amines. SpiroChem partnered with the University of Strasbourg to develop a palladium-catalyzed cross-coupling approach for the synthesis of $\mathrm{N}$-aryl-oxetanylamine (Scheme 2). ${ }^{[9]}$ This method provides new fragments potentially useful in medicinal chemistry as amide bioisosteres. This methodology can be used for bioisosteric switch of $N$-phenylarylamide, a very common motif present in several marketed drugs (Iminatib, Dasatinib, Entrectinib, ...).

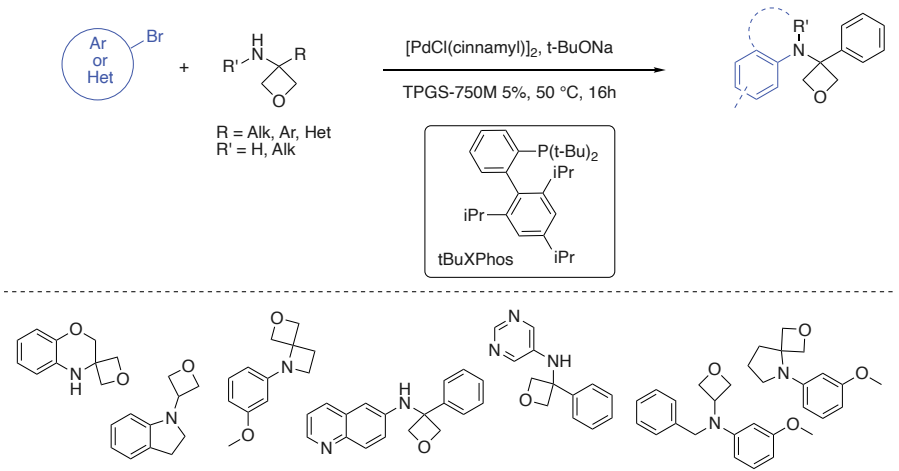

Scheme 2. N-Arylation of oxetanylamine.

Snapshot 3: As an alternative to palladium-catalyzed crosscouplings, metallaphotoredox catalysis is a powerful strategy to couple complex amines to heteroaromatics under relatively mild conditions. Taking advantage of our extensive library of spirocyclic and strained amines, valuable sp3-sp2 fragments were synthesized in parallel (Scheme 3). A high-performance blue LED photoreactor was used, allowing for ten reactions to be irradiated simultaneously, with short reaction times. Spirocyclic amines, fluorinated azetidines, and strained bicycloalkane-containing amines could be efficiently arylated with 3-bromo pyridine. This rapid screening also helped identifying problematic substrates, on which we can later focus our R\&D efforts. ${ }^{[10]}$

$$
\mathrm{R}^{1-\mathrm{N}} \mathrm{R}^{2} \stackrel{\begin{array}{l}
\text { cat. [Ni], cat. [lr]. cat. } \\
\text { (Het)Ar-Br, base }
\end{array}}{50^{\circ} \mathrm{C} \text {, blue LEDs }} \mathrm{R}^{1{ }^{1}-\mathrm{N}^{1}} \mathrm{R}^{2}
$$

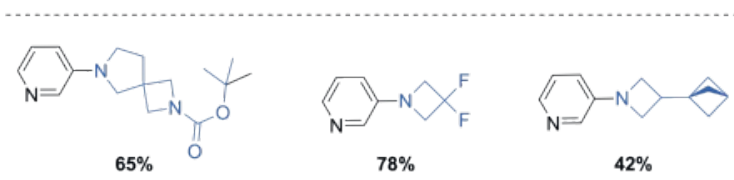

Scheme 3. Metallaphotoredox C-N coupling for fragment synthesis.

Snapshot 4: The rise of DNA-Encoded Libraries (DEL) created a need for elaborated building blocks with multiple orthogonal exit vectors. Given the recent advance of chemistry on DNA, it is important that the scaffold contain already all the structural information and functionalization vectors on a compact motif. In this example, we used simple blue light irradiation of a mixture of Boc-protected (aza)indoles and donor-acceptor diazo-compounds to generate a series of densely functionalized heterocyclic cores with three protected exit vectors: an amine, a carboxylic acid and an aryl halide (Scheme 4). The transformation was completely regio- and diastereoselective, giving valuable rigid sp2-sp3 building blocks. Selective deprotection of either the amine or the ester was possible, paving way for direct DEL applications. ${ }^{[11]}$

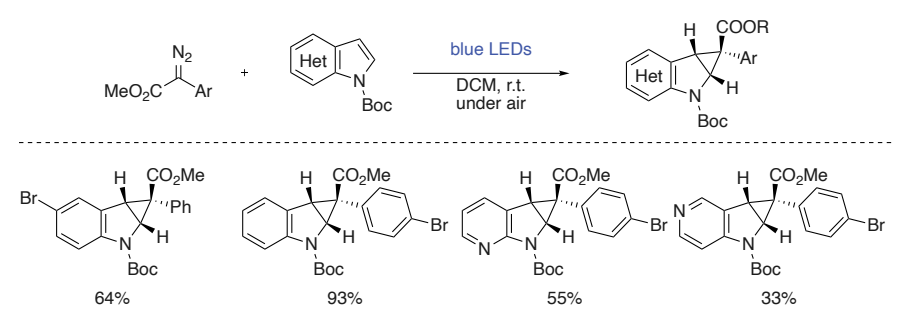

Scheme 4. Photoinduced cyclopropanes of indoles with diazo compounds.

Snapshot 5: Along with rapid generation of complex buildingblocks libraries, we also investigate late-stage functionalization of complex molecules with sp3-rich synthons such as bicycloalkanes. We have developed several privileged reagents for this purpose and we have developed accompanying methodologies to graft theme onto molecules of interest. For example, starting from SPC-a188, SPC-a728 could be synthesized in four steps. This robust dimethyldihydrooxazole derivative is a reagent of choice for Negishi cross-couplings. These reactions were successful under classic thermal conditions, and simultaneous irradiation with blue LEDs was in some cases beneficial. After coupling with an appropriate (hetero)aromatic halide, the carboxylic acid moiety could be unmasked by acidic hydrolysis, making further functionalizations straightforward (Scheme 5). ${ }^{[12]}$

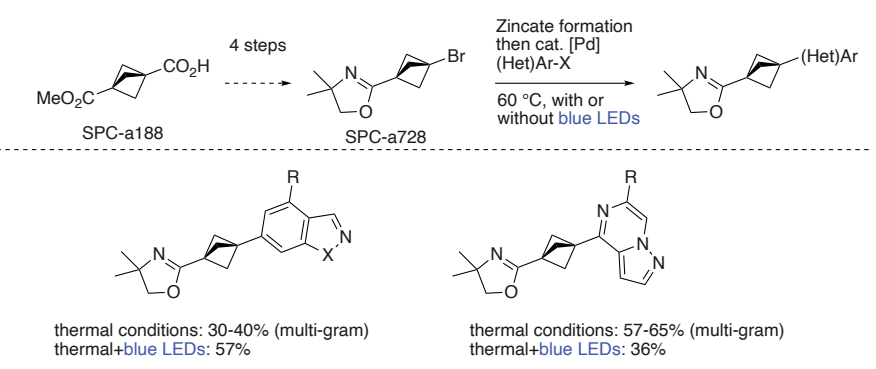

Scheme 5. The effect of light on Negishi coupling of bromobicycloalkanes with heteroaromatic halides. 
Snapshot 6: [1.1.1]-Propellane is another privileged reagent, easily accessed on multigram-scale. Recently, Japan Tobacco Inc published a radical multicomponent carboamination of [1.1.1]-propellane with hydrazyl reagents as a radical precursor and di-tert-butyl azodicarboxylate as a radical acceptor (Scheme 6). ${ }^{[13]}$ Hydrazines are easily accessed using literature procedures, which makes them privileged radical precursors. We successfully reproduced and expanded this methodology, but we also discovered that by omitting the di-tert-butyl azodicarboxylate, we could incorporate the bicyclopentane motif into complex molecules thereby providing an efficient strategy for their late-stage incorporation. The reaction was validated using phenyl hydrazine as model and 1-phenylbicyclo[1.1.1]pentane was obtained in good yield. Those conditions were transferred to a complex molecule (drug candidate).

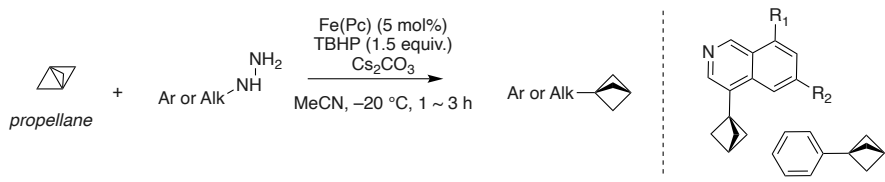

Scheme 6. Late stage incorporation of the bicyclopentane moiety.

\subsection{On the Importance of Operational Efficiency}

For all these discovery projects, we quickly realized that efficient benchtop analytical tools are of paramount importance for reaction analysis and can improve productivity by providing direct feedback on the success of parallel reactions. For example, reaction monitoring by TLC-MS enables rapid and precise assessment of the success of each parallel reaction. As seen in Fig. 3, a single TLC plate reporting five different reactions could be analyzed in less than five minutes, and the identity of the major product confirmed by mass. Combined with other bench-side techniques, such as Benchtop-NMR, ${ }^{[14]}$ precious time is saved during each experiment, which allows for rapid decision-taking and increased productivity.

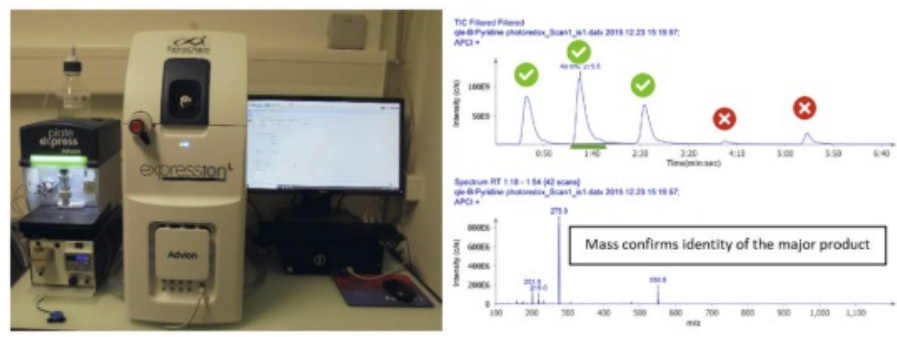

Fig. 3. Efficient reaction analysis using TLC-MS.

\section{Product/Service and Outlook}

Products: Our unique collections are designed to help scientists accelerate their drug discovery and lead optimization programs. SpiroChem product portfolio includes a large set of novel building blocks, SpiroKits, ${ }^{[2]}$ scaffolds for DEL and sp3-enriched fragments. SpiroChem's novel motifs are must-haves to generate IP-protected starting points for drug design. Our libraries are unique, the chemistry is tractable and easily expandable. All compounds were specifically designed using chemical informatics tools for further improvements in ADME properties or coverage of specific domains of structural/shape space.

Services: Beyond our product offering through our catalog and libraries (fragments, scaffolds), our team has developed undisputable capabilities in the design, synthesis and production of complex small organic molecules and offers assistance on a wide range of chemistry projects. Depending on the complexity of projects, we provide tailored solutions under FTE (Full-Time Equivalent) collaborations. For example, we provide full support to FBDD (Fragment-Based Drug Design) projects by rapid fragment evolution and exploration campaigns. Because we master the chemistry around all our products, we can rapidly generate analogues that will allow to explore the chemical space around fragment hits (Fig. 4).

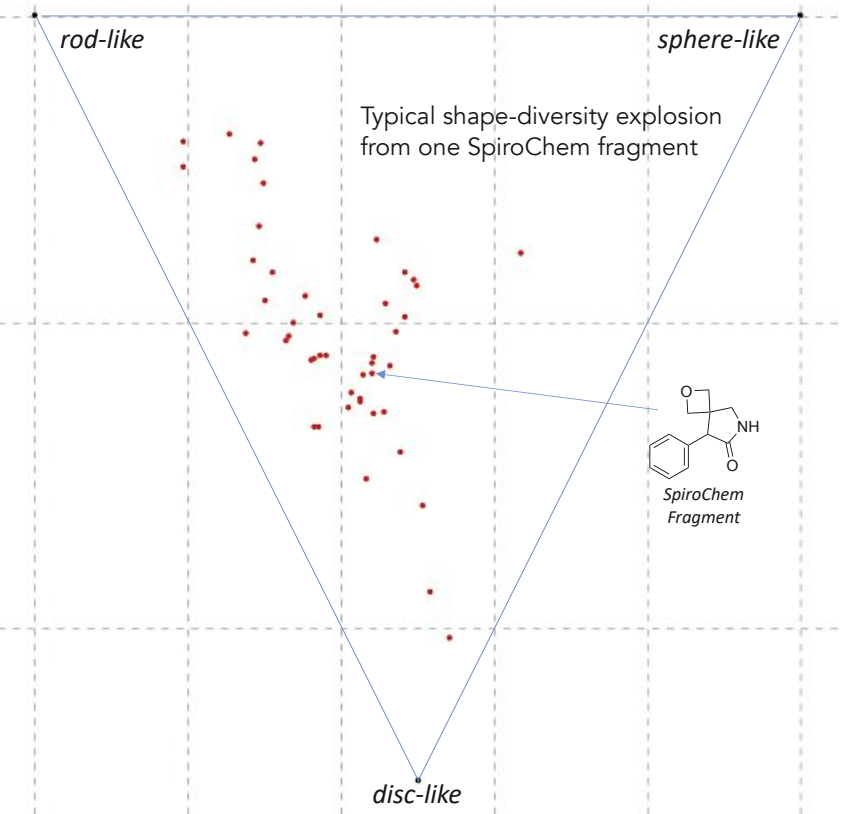

Fig. 4. Diversity generation by exploiting the accessible near chemical space.

We bring efficiency in drug-discovery projects from hit identification through lead generation and optimization to the selection of a drug candidate.

Over the years, we have also expanded the span of our expertise and developed SpiroChem's top-quality medicinal chemistry services. Our broad expertise in bio-isosteric replacements for drug discovery projects, together with SpiroChem's portfolio of products, can allow an easy and fast access to novel and patentable structural space. Furthermore, our team can support needs of our clients by providing high-class route scouting and process optimization services which include flow and photo-flow chemistry.

Through innovation, SpiroChem is bringing back synthetic chemistry to the forefront of discovery and is developing a best-in class chemistry R\&D platform that provides solutions to accelerate medicinal chemistry projects. The dual Product/Service model is a strength and allows a cross-fertilization of business lines that accelerate R\&D. We also believe in a collaborative model for $\mathrm{R} \& D$, where a consortium of best-in-class service providers efficiently join forces to shorten the overall lead time for drug discovery. We facilitate access to our network of partners for our clients. 


\section{SpiroChem}

SpiroChem AG is an elite Swiss fine chemicals company specialized in the design, production and commercialization of novel building blocks for drug discovery. Our unique repertoire of molecules and associated synthetic tools was designed to accelerate drug discovery and lead optimization programs in Life Science. In particular, our products and services allow scientists and medicinal chemists to improve ADME properties, access new proprietary chemical space and design better drugs for tomorrow. SpiroChem is recognized as the world-leading company on the concept of bio-isosterism and conformationally-restricted molecules.

Our cutting-edge know-how in chemistry has a track record of success: SpiroChem is the ideal collaboration partner for life science companies seeking to strengthen their outsourcing strategy with an innovation partner.

SpiroChem's FTE solutions include medicinal chemistry, enabling chemistry platform for efficient structural space exploration and efficient route scouting.

We deliver solutions, not just services!

\section{Key Figures}

Founding Year 2011

Location(s)

Basel, Switzerland

Legal Form AG

Founders

Scientific Advisors

Thomas C. Fessard

Erick M. Carreira

Peter Harboe-Schmidt

\begin{tabular}{l|l} 
& Prof. Dr. Carreira \\
& Prof. Dr. Sarlah \\
& Dr. Marc Lang
\end{tabular}

No of employees

50

\section{Key Inventions}

Manufacturing process

\section{Next Milestones}

Q4 2021

Q4 2022
Efficient manufacturing process for the production of bicyclo[1.1.1] pentane molecules (patented)

File 2-3 additional patents on important motifs or methods for medicinal chemistry.

SpiroChem is constantly innovating and invests in the development of new products and synthetic tools, some of which turn out to be very valuable!

Deployment of AI/Machine-learning platform SpiroChem is committed to always push the boundaries of the accessible chemical space, and we have started an Innosuisse-sponsored collaboration with a team of expert in Machine Learning from HES-SO, Geneva, Switzerland
Awards (or other important information)

2012

IMD Start-up Award

2015

Venture Leaders China

\section{Contact}

Contact Person

Thomas C. Fessard, CEO

Address

SpiroChem AG

Mattenstrasse 24

4058 Basel, Switzerland

Email contact@spirochem.com

Website www.spirochem.com

\section{Acknowledgements}

The authors thank the entire team of passionate chemistry experts at SpiroChem, their outside collaborators and their advisors for their hard work and daily commitment to push further the frontiers of chemistry.

Received: August 31, 2020

[1] a) F. Lovering, J. Bikker, C. Humblet, J. Med. Chem. 2009, 52, 6752; b) C. P. Tinworth, R. J. Young, J. Med. Chem. 2020, 63, 10091

[2] www.spirochem.com/spirokits

[3] Innosuisse grant 46966.1 IP-ICT (2020-2022)

[4] www.ChemAlive.com

[5] a) A. F. Stepan, C. Subramanyam, I. V. Efremov, J. K. Dutra, T. J. O'Sullivan, K. J. DiRico, W. S. McDonald, A. Won, P. H. Dorff, C. E. Nolan, S. L. Becker, L. R. Pustilnik, D. R. Riddell, G. W. Kauffman, B. L. Kormos, L. Zhang, Y. Lu, S. H. Capetta, M. E. Green, K. Karki, E. Sibley, K. P. Atchison, A. J. Hallgren, C. E. Oborski, A. E. Robshaw, B. Sneed, C. J. O'Donnell, J. Med. Chem. 2012, 55, 3414; b) K. C. Nicolaou, D. Vourloumis, S. Totokotsopoulos, A. Papakyriakou, H. Karsunky, H. Fernando, J. Gavrilyuk, D. Webb, A. F. Stepan, ChemMedChem 2016, 11, 31 .

[6] I. S. Makarov, C. E. Brocklehurst, K. Karaghiosoff, G. Koch, P. Knochel, Angew. Chem. Int. Ed. 2017, 56, 12774.

[7] S. J. Rupert, J. P. Proctor, Angew. Chem. Int. Ed. 2019, 58, 13666.

[8] a) J. A. Burkhard, G. Wuitschik, M. Rogers-Evans, K. Müller, E. M. Carreira, Angew. Chem. Int. Ed. 2010, 49, 9052; b) M. McLaughlin, R. Yazaki, T. C. Fessard, E. M. Carreira, Org. Lett. 2014, 16, 4070.

[9] M. Bollenbach, W. Lecroq, P. Wagner, T. Fessard, M. Schmitt, C. Salome, Chem. Comm. 2019, 55, 1623.

[10] a) E. B. Corcoran, M. T. Pirnot, S. Lin, S. D. Dreher, D. A. DiRocco, I. W. Davies, S. L. Buchwald, D. W. C. MacMillan, Science 2016, 353, 279; b) Q. Lefebvre, C. Salomé, T. C. Fessard, Beilstein J. Org. Chem. 2020, 16, 982.

[11] I. D. Jurberg, H. M. L. Davies, Chem. Sci. 2018, 9, 5112.

[12] a) I. Abdiaj, A. Fontana, M. V. Gomez, A. de la Hoz, J. Alcázar, Angew. Chem. Int. Ed. 2018, 57, 8473; b) I. Abdiaj, L. Huck, J. M. Mateo, A. de la Hoz, M. V. Gomez, A. Díaz-Ortiz, J. Alcázar, Angew. Chem. Int. Ed. 2018, $57,13231$.

[13] J. Kanazawa, K. Maeda, M. Uchiyama, J. Am. Chem. Soc. 2017, 139, 49, 17791.

[14] www.nanalysis.com

\section{License and Terms}

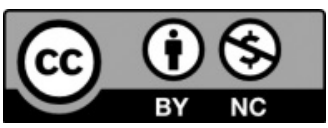

This is an Open Access article under the terms of the Creative Commons Attribution License CC BY_NC 4.0. The material may not be used for commercial purposes.

The license is subject to the CHIMIA terms and conditions: (http:// chimia.ch/component/sppagebuilder/?view=page \&id=12).

The definitive version of this article is the electronic one that can be found at https://doi.org/10.2533/chimia.2020.803 